\title{
STRANGENESS PRODUCTION IN MINIMUM-BIAS EVENTS AT CDF
}

\author{
N. MOGGI, for the CDF collaboration \\ University and I.N.F.N., Bologna \\ viale B. Pichat $6 / 2$ \\ 40127 Bologna, ITALY \\ e-mail: moggi@bo.infn.it
}

\section{Introduction}

In a recently published paper ${ }^{1}$ are emphasized, for the soft subsample extracted from the minimum-bias (MB) dataset of $p \bar{p}$ collisions, interesting invariances with the c.m. energy of the charged multiplicity and $p_{T}$ distributions. We present an analogous study on $V^{0}$ production.

\section{Data Set and Event Selection}

Data were collected with a MB trigger at $\sqrt{s}=1800$ and $630 \mathrm{GeV}$ by the CDF detector at the Tevatron Collider during RunI. The CDF apparatus has been described elsewhere ${ }^{2}$. The analysis uses charged tracks reconstructed within the Central Tracking Chamber (CTC). The transverse energy flux was measured by the calorimeter system covering from -4.2 to 4.2 in $\eta$. Offline selection removed events with more than one primary vertex and with symptoms of known calorimeter problems. After all event selection cuts, 2,079,154 events remain in the full MB sample at $1800 \mathrm{GeV}$, and 2,091,599 in that at $630 \mathrm{GeV}$.

The selection of Soft and Hard interactions is described in detail in ${ }^{1}$.

\section{Selection of primary tracks, $K_{s}^{0}$ and $\Lambda^{0}$}

Reconstructed tracks within each event must pass selection criteria designed to remove the main sources of background. Accepting only tracks with $p_{T} \geq 0.4 \mathrm{GeV} / \mathrm{c}$ and within $|\eta| \leq 1.0$ ensures full efficiency and acceptance.

$V^{0}$ 's are selected from the full sample of the CTC reconstructed tracks, looking for unlike charge pairs converging to a common secondary vertex. 
A 3-constraint fit is performed for the two tracks coming from the secondary vertex with the hypothesys of $K_{s}^{0}$ or $\Lambda^{0}$ decay. We requires that:

- $L_{x y} \geq 1.0 \mathrm{~cm}$ (where $L_{x y}$ is the secondary vertex displacement in the $x-y$ plane);

- decayed tracks are within $|\eta| \leq 1.5$ and $p_{T} \geq 0.250 \mathrm{GeV} / \mathrm{c}$;

- the $V^{0}$ line of flight is close to the event vertex along the $z$ axis so that: $\mid Z_{0}^{V^{0}}$ pseudo-track $-Z^{\text {vertex }} \mid<6 \mathrm{~cm}$;

- $p_{T}\left(V^{0}\right)>0.4 \mathrm{GeV} / \mathrm{c}$ and $\left|\eta\left(V^{0}\right)\right|<1.5$;

The invariant mass distributions after all cuts shows that virtually no background is left in our $V^{0}$ sample.

After all selection cuts we find in the $1800 \mathrm{GeV}$ MB sample $57,787 K_{s}^{0}$ and $15,487 \Lambda^{0}$; in the $630 \mathrm{GeV}$ MB sample $54,581 K_{s}^{0}$ and $12,596 \Lambda^{0}$. Of all $K_{s}^{0}$, the fraction found in the soft sample is $\sim 20 \%$ and $\sim 32 \%$ at 1800 and $630 \mathrm{GeV}$ respectively $\left(\sim 13 \%\right.$ and $\sim 22 \%$ respectively for $\left.\Lambda^{0}\right)$.

\section{Results and Discussion}

Figure 1 shows the multiplicity distributions of $K_{s}^{0}$ and $\Lambda^{0}$ for the full $\mathrm{MB}$, the soft and the hard samples. All data are corrected for detection
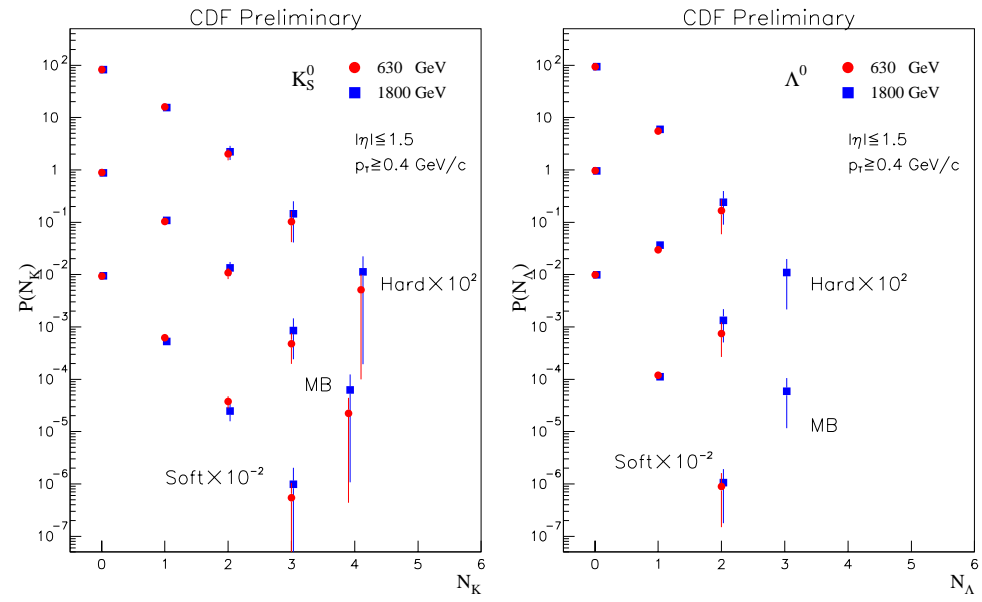

Figure 1. $\quad K_{s}^{0}$ and $\Lambda_{s}^{0}$ multiplicity distributions at 1800 and $630 \mathrm{GeV}$.

and reconstruction efficiency. The MB inclusive $p_{T}$ distributions of $K_{s}^{0}$ are shown in Fig. 2. Distributions of the soft subsample are also shown (because of space limitation we can't show also the hard subsample and all 
the distributions of $\Lambda^{0}$ ). Data are normalized to the number of events in each sample. Production rates and $p_{T}$ distributions are in good agreement with previous MB measurements ${ }^{3}$; this analysis is extended to higher $p_{T}$ values. The dependence of the average $K_{s}^{0} / \Lambda^{0} p_{T}$ on $N_{c h}^{*}$ is shown in
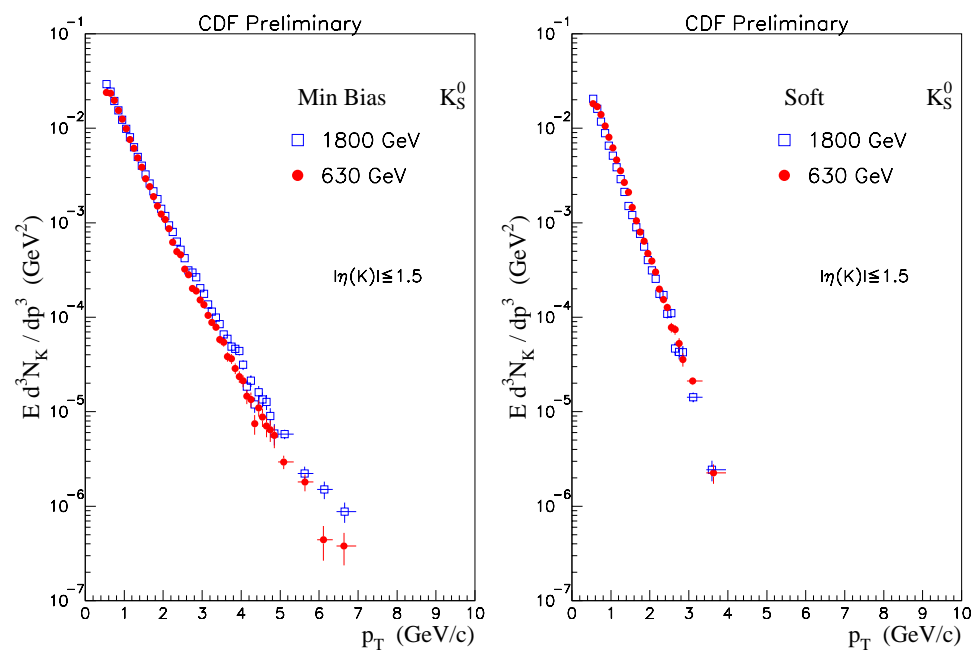

Figure 2. $\quad K_{s}^{0}$ inclusive $p_{T}$ distributions for the full MB and the soft samples

Fig. 3. As it is mentioned in ${ }^{4}$, it can be observed to increase with a slope roughly equal or greater than for charged particles, which are mainly pions (compare with ${ }^{1}$ ). There is no clear indication of a different energy dependence of the soft subsample with respect to MB and hard data but it may be interesting to notice that the slope of the correlation remains positive in the soft subsample which is highly depleted in high $E_{T}$ events. Finally, the dependence of the mean number of $K_{s}^{0} / \Lambda^{0}$ produced on the event charged multiplicity is presented in Fig. 4 where the ratio of the average number of $K_{s}^{0} / \Lambda^{0}$ to the event charged multiplicity $N_{c h}^{*}$ is plotted versus $N_{c h}^{*}$. The dependence is more pronounced for hard than for soft data and seems to flatten at larger multiplicities.

\section{Conclusions}

Higher statistics and accurate efficiency corrections give good improvement to the existing data of strange particle production in MB proton-antiproton interactions. Completely new is the measure of $K_{s}^{0}$ and $\Lambda^{0}$ production in the soft and hard subsamples. 

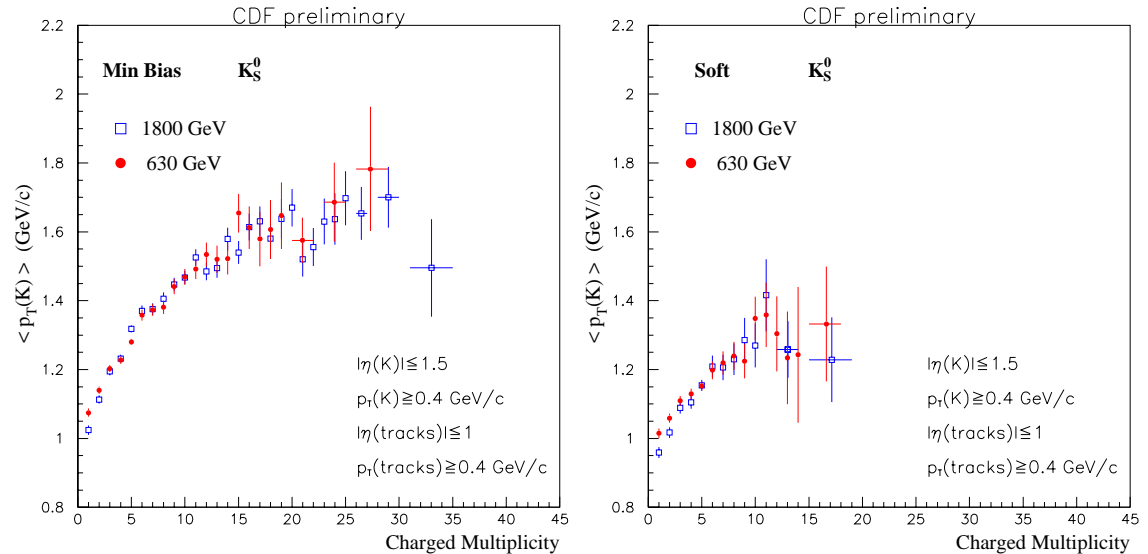

Figure 3. The average $p_{T}$ of $K_{s}^{0}$ as a function of the event charged multiplicity for the full MB and the soft samples at 1800 and $630 \mathrm{GeV}$.
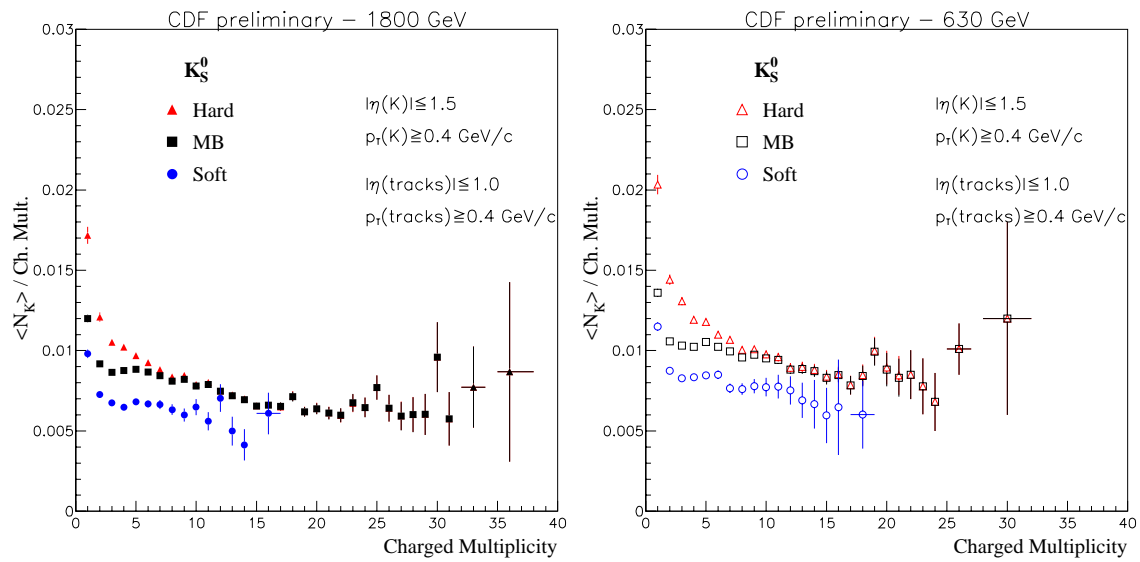

Figure 4. Average number of $K_{s}^{0}$ and produced in events of equal charged multiplicity $\left(N_{c h}^{*}\right)$ divided by the multiplicity itself and plotted vs the multiplicity.

\section{References}

1. D.Acosta et al., Phys. Rev. D65, 072005 (2002);

2. F.Abe et al., Nucl. Instrum. Methods A 271, 387 (1988), and references therein.

3. F.Abe et al., Phys. Rev. D40, 3791 (1989);

4. S.Banerjee et al., E735 Collaboration, Phys. Rev. Lett 62, 12 (1989); T.Alexopoulus, II Int.Conf. on Phys. and Astrophys. of Quark-Gluon Plasma,Calcutta,Jan.1993. 